\title{
DEVELOPMENT OF A BEE FEEDING PROCEDURE TO SUPPORT BEE HEALTH PROTECTION
}

Szabolcs Molnár - Ákos Juhász - Helga Szalontai

\section{Summary}

This study was conducted to investigate the effect of inulin (a commercial prebiotic) on the gut microbiota of the honeybee (Apis mellifera carnica pannonica). Some colonies of equal strength were fed with sugar syrup or inulin supplemented syrup at different dosages. Bee samples were collected at the beginning of the experiment and then every two weeks until the end of the study. The intestinal tracts of five bees per treatment were isolated, homogenised, diluted and the amount of living microbes (mainly the probiotic microbes) were determined by using differential and semi selective agar plates. Four different experiments were carried out in 2018 and 2019 and we found that the inulin has no detectable effect on the composition of gut microbiome by culture based methods in the case of LAB (lactic acid bacteria), Bifidobacterium spp, Snodgrassella, Gilliamella and Frischella sp.

Keywords: Honeybee, prebiotics, inulin, intestinal microbiota 


\section{Introduction}

The honeybee (Apis mellifera), as an important pollinator, contributes significantly to agricultural productions and the human food supply. Nowadays, bees are stressed by a number of biotic and abiotic factors (pathogens, pesticides, climate change, habitat loss, etc.) which affect health and productivity (Porrini et al., 2016; Alberoni et al., 2016) and persistent population decline has been reported several times over the last decade (Lee et al., 2015; Zee et al., 2012; Goulson et al., 2015). The European Union ban of antibiotics as feed additives has stimulated the search for natural alternatives (European Commission, 2005). Animals (as well as humans) have symbiotic microorganisms, which are important for the proper functioning of the gastrointestinal tract (for nutrition and pathogen defence). The intestinal system of the honeybee contains relatively few bacteria in number and variety. Eight major bacterial groups concentrated in the hindgut: two Alpha-, one Beta-and two Gammaproteobacteria, two closely related Lactobacillus groups (Firm 4, 5), and one Bifidobacterium (Moran, 2015). Some of them are found in all honey bees (Lactobacilli, Beta- and Gammaproteobacteria), but the other groups of bacteria may be completely absent from some animals/individuals (Moran et al., 2012). The most prominent Gram-negative members of the intestinal microbial community Snodgrassella alvi and Gilliamella apicola are mutualistic symbionts and appear to be unique to Apis (honeybee) and Bombus spp.(bumble bee). Frischella perrara and Bartonella apis are even more host-specific, occurring only in Apis spp (Moran et al., 2012; Kwong et al., 2017).

The link between intestinal microbiota and health status, have encouraged the researchers to use beneficial gut microorganisms and prebiotics to improve the health of animals (and humans). Probiotics (mainly lactic acid bacteria) and prebiotics (like inulin) help to protect the intestinal microbial health and are recommended to be added into the forage of different vertebrates and invertebrates. There are commercial probiotics and/or prebiotics containing diet supplements in beekeeping management too. The first documented application of probiotics in the case of honeybees occurred in 1997 (Machova et al., 1997). Since then, only a few studies have reported the use of pre/probiotics, and the effect was not always clear. Some data has shown that the use of probiotics and prebiotics has a beneficial effect on the bees and increases honey production (Patruica and Mot 2012; Patruica and Hutu 2013) whereas the others suggest that commercial probiotics increases honeybee mortality because most of them have been developed for humans and the differences between the gut microbiota between honeybees and vertebrates may cause an opposite effect (Andrearczyk et al. 2014; Ptaszynska et al., 2016).

We decided to study the effect of inulin, a well-known commercial prebiotic, on the honeybee and the changes of intestinal microbiome were studied by determining the number of potentially beneficial bacteria. 


\section{Material and methods}

In our experiment, the so-called "maintenance feeding" used by beekeepers was used as stimulant feeding and this sucrose syrup contains $2 \mathrm{~kg}$ sugar in 3 litre water. The selected honeybee colonies were healthy and equal in strength. The control group was fed with one litre sucrose syrup every second day. In the case of the treated group, it was the same but 30 or $60 \mathrm{~g} \mathrm{l}^{-1}$ was added to the syrup. The treatment was performed on three colonies in both cases and the samples (approximately 20 worker bees each) were collected before the treatment and 2, 4 and 6 weeks later, depending on the duration of the treatment. In later experiments we used an absolute control (without any treatment) to analyse the external, internal and environmental impacts. The intestinal tracts of five bees were isolated, homogenised and diluted in $1 \%$ tripton containing saline. Traditional microbiological methods were used to determine the amount of living microbes. Five different media (Nutrient agar, De Man, Rogosa and Sharpe agar, Eosin Methylene Blue agar, Columbia Blood agar, and Bifidobacterium medium) were used to investigate total aerobic bacteria; Lactobacillus; coliform bacteria; Snodgrassella, Gilliamella and Frischella; Bifidobacterium, respectively. The inoculated agar plates were incubated at $37 \pm 2{ }^{\circ} \mathrm{C}$ for $48-72$ hours in ambient air or in anaerobic (in the case of Frischella and Bifidobacterium) and microaerophilic conditions (5\% CO in the case of Lactobacillus, Snodgrassella and Gilliamella). The blood agar was neither selective nor differential to Snodgrassella, Gilliamella and Frischella; further basic biochemical tests and microscopic examinations were applied to confirm the strains identity. The amount of bacteria was calculated per gram of intestine $\left(\mathrm{CFU} \mathrm{g} \mathrm{g}^{-1}\right)$.

\section{Results}

We have compared the repeatability of sample processing in the case of one and five animals. The complete intestinal system was isolated, homogenised and the colony numbers of bacteria was normalised to one gut. In the case of individual isolates, the deviation is very high, especially in the case of coliform bacteria $\left(2.0 \times 10^{2}\right.$ $\left.1.3 \times 10^{7}\right)$. We found that the difference between the parallel samples containing five homogenized gut per sample was smaller than for single intestinal samples and the pooling of five intestines gives a more reproducible result. Apart from the differences in the individual animals, the individual honeybee colonies also differed significantly, so the results obtained for several hives were not comparable. The literature also confirms that there are very large differences between the intestinal microbes of individual animals in the case of the honeybee (Moran et al., 2012). 
In later experiments, based on our results, the exact colony count was not compared, but only the tendency of changes due to different treatments was calculated. The bees were treated with sugar syrup and then with increasing inulin concentration. We found that the amount of certain bacteria (total aerobic bacteria and Lactobacillus) increased significantly (ten to one hundredfold) as a result of the sugar syrup treatment. The effect of the inulin was not detectable: only the amount of aerobic bacteria changed significantly, the beneficial microbes were not affected. We think that these changes are due not to the treatments but to differences between individual animals.

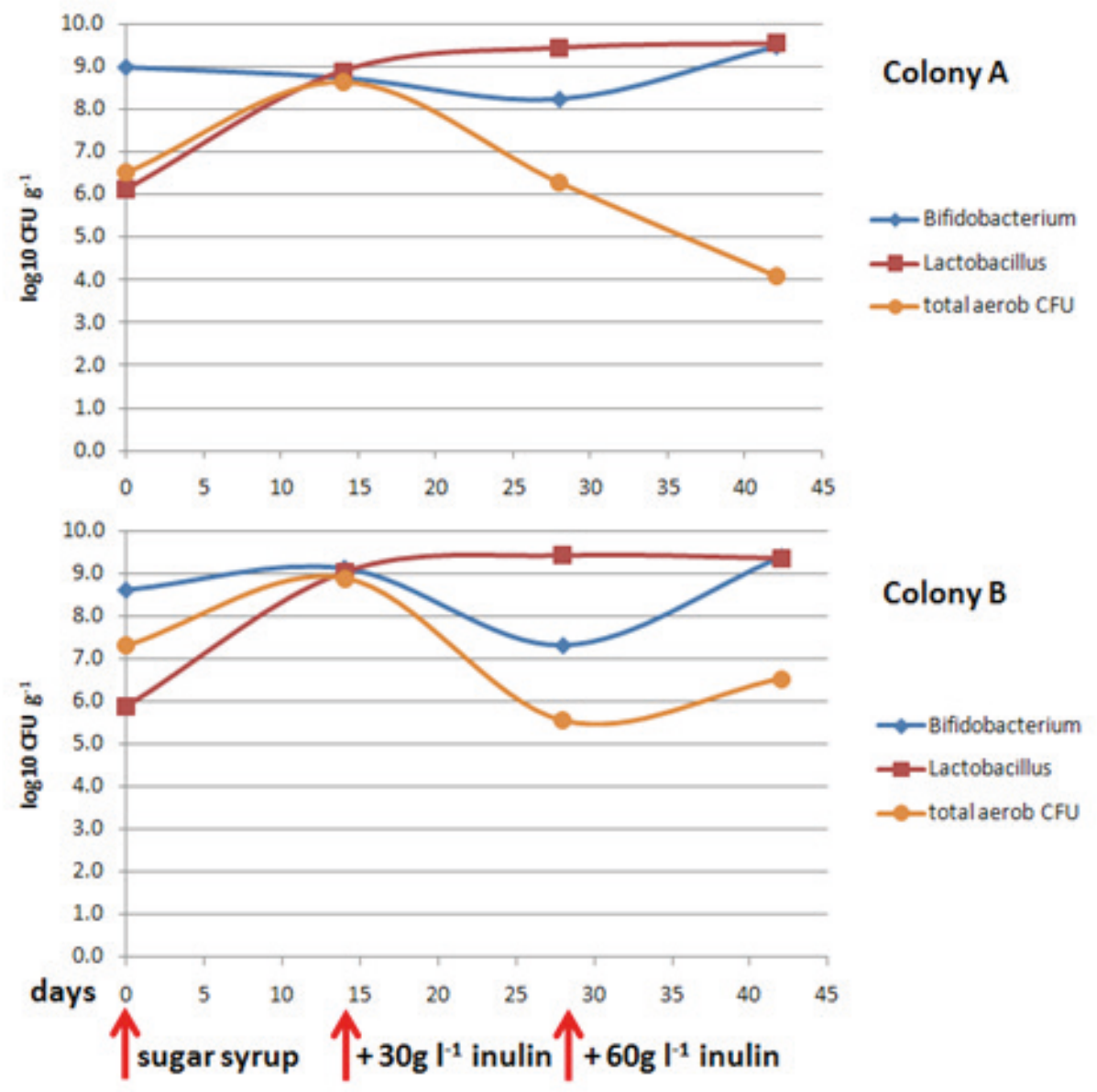

Fig. 1.: The effect of inulin on the intestinal microbiota of two honeybee colonies. 
In the following experiment the amount of Snodgrassella, Gilliamella and Frischella were also determined. The treated group received the same treatment as before but the control group was not treated at all, not even with sugar syrup. We found that the inulin treatment did not significantly influence the amount of these bacteria.

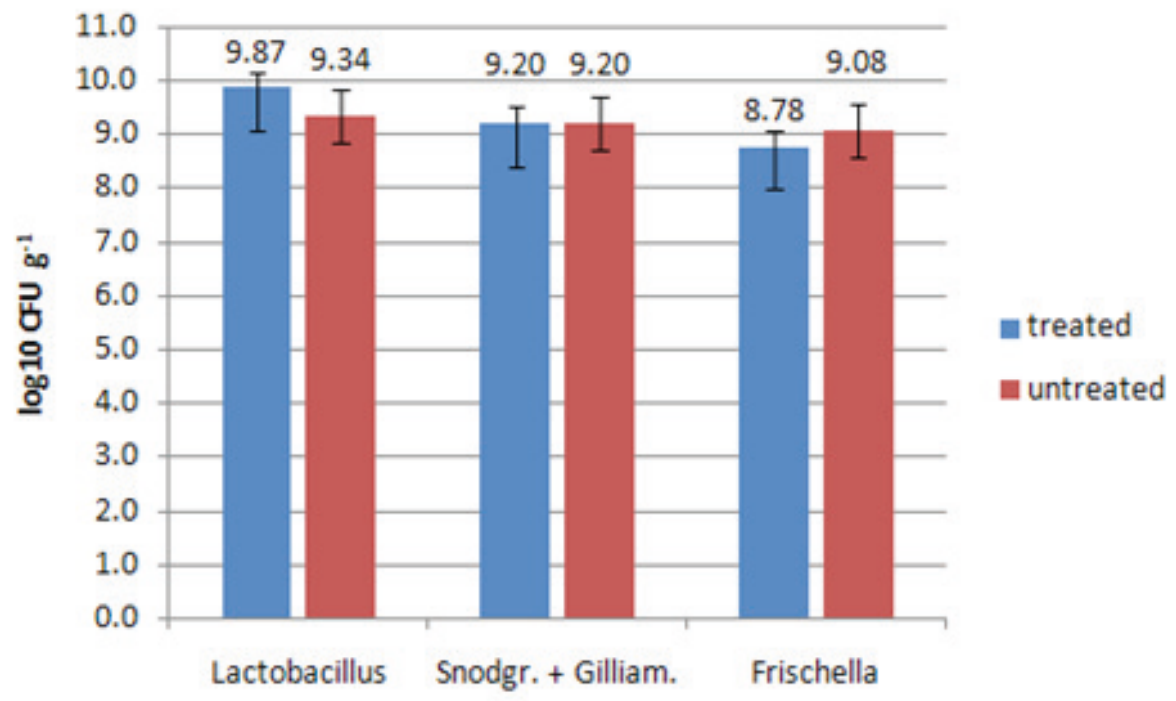

Fig. 2.: The amount of Lactobacillus, Snodgrassella, Gilliamella and Frischella $\left(\log 10 \mathrm{CFU} \mathrm{g}{ }^{-1}\right)$ in the end of the experiment. The treated group was fed with inulin supplemented sugar syrup and the untreated group was an absolute control (without any treatment).

\section{Conclusions}

We have developed a method that can be used to test the changes of gut microbiota in the case of the honeybee. The prebiotic effect of inulin was not detected by the presently used technique. This result supports the hypothesis that commercially available pre- and probiotics may not be applied uniformly to all groups of animals.

\section{Acknowledgement}

This research was supported by the grant EFOP-3.6.1-16-2016-00001 and 2017-1.3.1VKE-2017-00001. 


\section{References}

[1.] Porrini, C. - Mutinelli, F. - Bortolotti, L. - Granato, A. - Laurenson, L. Roberts, K. - Gallina, A. - Silvester, N. - Medrzycki, P. - Renzi, T. - Sgolastra, F. - Lodesani, M. (2016): The status of honey bee health in Italy: results from the nationwide bee monitoring network. PLoS One 11: 5. e0155411. doi:10.1371/journal.pone.0155411

https://doi.org/10.1371/journal.pone.0155411

[2.] Alberoni, D. - Gaggia, F. - Baffoni, L. - Di Gioia, D. (2016): Beneficial microorganisms for honey bees: problems and progresses. Applied Microbiology and Biotechnology. 100: 9469-9482.

https://doi.org/10.1007/s00253-016-7870-4

[3.] Lee, K.V. - Steinhauer, N. - Rennich, K. - Wilson, M.E. - Tarpy. D.R. Caron D.M. et al. (2015): A national survey of managed honey bee 20132014 annual colony losses in the USA. Apidologie. 46: 292-305..

https://doi.org/10.1007/s13592-015-0356-z

[4.] Zee, R. - van der Pisa. L. - Andonov. S. - Brodschneider, R. - Charrière, J.D. - Chlebo, R. et al. (2012): Managed honey bee colony losses in Canada, China, Europe, Israel and Turkey, for the winters of 2008-9 and 2009-10. J. Apic. Res. 51: 100-14.

https://doi.org/10.3896/IBRA.1.51.1.12

[5.] Goulson, D. - Nicholls, E. - Botías, C. - Rotheray, E.L. (2015): Bee declines driven by combined stress from parasites, pesticides, and lack of flowers. Science. 347: 1255957.

https://doi.org/10.1126/science. 1255957

[6.] European Commission. (2005): Ban on antibiotics as growth promoters in animal feed enters into effect. http://europa.eu/rapid/press-release_IP-051687_en.htm

[7.] Moran, N.A. (2015): Genomics of the honey bee microbiome. Current Opinion in Insect Science 10: 22-28.

https://doi.org/10.1016/j.cois.2015.04.003

[8.] Moran, N.A. - Hansen, A.K. - Powell, J.E. - Sabree, Z.L. (2012): Distinctive gut microbiota of honey bees assessed using deep sampling from individual worker bees. PLoS ONE 7: 4. e36393.

https://doi.org/10.1371/journal.pone.0036393 
[9.] Kwong, K.W. - Medina, L.A. - Koch, H. - Sing, K-W. - Soh, E.J.Y. - Ascher, J.S. - Jaffe, R. - Moran, N.A. (2017): Dinamic microbiome evolution in social bees. Sci. Adv. 3: e1600513.

https://doi.org/10.1126/sciadv.1600513

[10.] Machova, M - Rada, V - Huk, J - Smekal, F. (1997): Entwicklung der bienenprobiotik. Apiacta 32: 99-111.

[11.] Patruica, S. - Mot, D. (2012): The effect of using prebiotic and probiotic products on intestinal micro-flora of the honeybee (Apis mellifera carpatica). Bull. Entomol. Res. 102: 619-623.

https://doi.org/10.1017/S0007485312000144

[12.] Patruica, S. - Hutu, I. (2013): Economic benefits of using prebiotic and probiotic products as supplements in stimulation feeds administered to bee colonies. Turk. J. Vet. Anim. Sci. 37: 259-263.

[13.] Andrearczyk, S. - Kadhim, M.J. - Knaga, S. (2014): Influence of a probiotic on mortality, sugar syrup ingestion and infection of honeybees with Nosema spp. under laboratory assessment. Med. Weter. 70: 762-765.

[14.] Ptaszynska, A.A. - Borsuk, G. - Zdybicka-Barabas, A. - Cytrynska, M. Małek, W. (2016): Are commercial probiotics and prebiotics effective in the treatment and prevention of honeybee nosemosis C? Parasitology Research 115: 397-406. doi:10.1007/s00436-015-4761-z

https://doi.org/10.1007/s00436-015-4761-z

\section{Authors:}

\section{Juhász Ákos Ph.D.}

Szent István University, Institute of Genetics, Microbiology and Biotechnology, Department of Microbiology and Environmental Toxicology e-mail: ajuhasz11@gmail.com

\section{Molnár Szabolcs}

Eszterházy Károly University, Centre for Research and Development, Food and Wine Research Institute e-mail: molnarszabolcs.hun@gmail.com

\section{Szalontai Helga Ph.D.}

Eszterházy Károly University, Centre for Research and Development, Food and Wine Research Institute e-mail: szalontai.helga@uni-eszterhazy.hu 